\title{
A Clinical Research about the Intermittent Medication Treatment on the Female Urogenital Tract Mycoplasma Infection
}

\author{
Guosheng Su1, Xiaolu Luo1*, Rongwei Liang², Lianhua Pan'1 \\ ${ }^{1}$ The Fourth People's Hospital, Nanning, China \\ ${ }^{2}$ Maternal and Child Health Care Hospital, Qinzhou, China \\ Email: "luxiaolu2007@126.com
}

Received 20 July 2014; revised 15 August 2014; accepted 11 September 2014

Copyright (C) 2014 by authors and Scientific Research Publishing Inc.

This work is licensed under the Creative Commons Attribution International License (CC BY). http://creativecommons.org/licenses/by/4.0/

(c) (i) Open Access

\begin{abstract}
Object: To explore a better treatment on the female urogenital tract mycoplasma infection so as to ensure the health of female reproduction. Method: 115 female patients with urogenital tract mycoplasma infection are selected, including 63 patients acted as a group for intermittent medication treatment; that is to say, the ones that are confirmed to be sensitive drugs to those 63 patients are chosen, and let them take this medication for 15 days and stop taking it for 7 days and after that continue to take it for 10 days. The other 52 patients in the other group which acts as a contrast to the former group are cured with a course of conventional treatment for $15-21$ days. Results: As to those 115 female patients with urogenital tract mycoplasma infection, $\mathbf{4 3}$ patients get relapsed, and 27 patients of them are in the contrast group and 16 patients are in the treatment group. There is obvious difference on the contrast of relapse rate between the treatment group and contrast group, and they are $P<0.01$. But as to the sensitive testing results of 10 common antibiotic medicines, there are cases of illness of relapse in those 2 groups, and the rate of drug sensitivity in the treatment group is higher than that of the contrast group. Between treatment group and contrast group, there is obvious difference in the sensitivity of the drugs, $P<0.05$ average. Conclusion: When the female patients with urogenital tract mycoplasma infection are cured with a traditional treatment, because of the side effects of drugs, they are not likely to take medicines that are required to making the cure rate become lower and the relapse rate become higher. But intermittent medication is designed with the half-life period of medicine, which makes the medicine can be digested and absorbed easily, and the cure rate is high and the relapse rate is low. With this research we found a more effective method of the treatment on the female urogenital tract mycoplasma infection and showed the clinical significance in intermittent medication.
\end{abstract}

\footnotetext{
"Corresponding author.
} 


\section{Keywords}

\section{Intermittent, Urogenital Tract, Mycoplasma, Drug Sensitivity}

\section{Introduction}

Nowadays, with the development of social economy and the deepening of reform and opening up, the infection rate of sexually transmitted diseases is becoming increasingly high. In addition, mycoplasma is also an important part in the five STD of human beings. Especially the female urogenital tract owns the opening characteristic of biorgan and it is likely to be infectious, and this kind of problem brings great puzzles to human's lives. Currently the bacterium sex urethritis, sterility and infertility are the 2 STD that are of high morbidity, and the main reasons for them are the carelessness of hygiene in life, unclean sexual behaviors and multiple sex partner [1] [2]. In order to explore a better treatment on the female urogenital tract mycoplasma infection to ensure their health, we obtain specific treatment effects after trail or errors. Here are the results as following.

\section{Clinical Data}

1) After the project was approved, director of this project called all of the team members to take some related training. Otherwise ask them do the research separately and corporately. The team members selected 115 cases of female genitourinary tract mycoplasma infection patients as the research objects from daily clinical work. And the samples of 115 female patients with urogenital tract mycoplasma infection come from the patients of outpatient service of obstetrics and gynecology in our hospital and the patients with hospitalization in January 2012 to June 2013. Those patients come to have treatment for some reasons, and they are micturition, urgent urination, dysuria, urethral grout, thin viscous purulent secretion, the increase of vaginal secretion, hypogastrium pain and sterility infertility. The disease development in the 115 cases of patients is nearly consistent. That is, both for genitourinary tract mycoplasma infection. And the 115 patients were randomly divided into two groups according to the doctor's drug habit artificially. The control group included 52 cases, and the treatment group was 63.

2) Culture medium and source of drugs mycoplasma double AGAR color and concentration of drug sensitive test reaction plate are provided by Lituo Development Company Ltd. in Zhuhai, Guangdong, China. All reagents are used within a short period and are controlled with requirements.

3) Culture medium and testing method of drug sensitivity. Firstly we use the sample injector to absorb the non-inoculate sample medium 1001 and inject them to the first hole of the row A of the wattle with cover, then insert the cotton swab with secretions into the nutrient solution, shake and wash, and wipe the cotton swab on the in wall of the bottle, and get 150 - 200 liquid sample or 50/ $\mu$ positive sample and shake it up with culture medium and mix the samples and medium mixture into microwells of row A, B respectively. Except A1 (note: the drug susceptibility of row A is low concentration peridium well, row B is high concentration peridium well), add 1 drop of mineral oil to cover all wells, then put the culture medium and micro-wattle under the constant temperature of $37^{\circ} \mathrm{C}$ and foster them between $20 \mathrm{~h}-24 \mathrm{~h}$ and observe the growing of mycoplasma urealytium. And observe the growing of mycoplasma hominis between $36 \mathrm{~h}-48 \mathrm{~h}$ and record the results of them.

4) Comparison of methods and judgment if the every micro-well becomes muddy, and it indicates that there are pollutions, and the result should not be reported. If every micro-well turns yellow to red and it is clear, and it indicates that mycoplasma or something like that grow, and they are expressed with "+”, and the ones with no color changes show that mycoplasma or something like that don't grow, and they are expressed with “-”. $(+)=$ red, $(-)=$ yellow. The result of mycoplasma urealytium is reported between $20 \mathrm{~h}-24 \mathrm{~h}$, and the result of mycoplasma hominis is reported between $36 \mathrm{~h}-48 \mathrm{~h}$. 1) A1 is negative contrast, which should be yellow, if it is red, and it means that the failure of culture medium. 2) A2(-) indicates that the mycoplasma don't grow, A2(+) indicates that the mycoplasma grow $\mathrm{Uu}-\mathrm{Mh}<10^{4}$. 3) A2(+)B1(+) mycoplasma urealytium $\left(\mathrm{Uu} \geq 10^{4}\right)$. 4) $\mathrm{A} 2(+)$ $\mathrm{B} 2(+)$ mycoplasma hominis grow $\left(\mathrm{Mh} \geq 10^{4}\right)$. 5) A3-A12, B3-B12 express the concentration of antibiotic, the results of drug sensitivity are the following: $\mathrm{A}(-) \mathrm{B}(-)$ are sensitive, $\mathrm{A}(+) \mathrm{B}(-)$ are medium sensitive, $\mathrm{A}(+) \mathrm{B}(+)$ are resistant. 
5) Therapeutic method: select 115 female patients with urogenital tract mycoplasma infection, including 63 patients acted as a group for intermittent medication treatment, that is to say, choose the ones that are confirmed to be sensitive drugs to those 63 patients, and let them take this medication for 15 days and stop taking it for 7 days and after that continue to take it for 10 days. The other 52 patients in the other group which acts as a contrast to the former group are cured with a course of conventional treatment for 15 - 21 days.

6) After the 2 groups took those medications, then to have the mycoplasma culture on them a week later. If the group which is for contrast are rechecked to be positive, then it is called the contrast group relapse, then this group is regarded as treatment group for treatment until they are cured. After the treatment for the treatment group, if the mycoplasma is still positive, then it is called treatment group relapse.

7) Statistical analysis use the software SPSS17.0 for statistical analysis and measurement data is expressed with $\bar{X} \pm \mathrm{S}$. The comparisons of the number of cases of relapse of the two groups are tested with $X^{2}$, and there are statistical significance with the difference of $\mathrm{P}<0.05$.

\section{Results}

As to those 115 female patients with urogenital tract mycoplasma infection, 43 patients get relapsed, and 27 patients of them are in the contrast group and 16 patients are in the treatment group. There is obvious difference on the contrast of relapse rate between the treatment group and contrast group, and they are $X^{2}=8.5623, \mathrm{P}<0.01$. But as to the sensitive testing results of 10 common antibiotic medicines, there are cases of illness of relapse in those two groups, and the rate of drug sensitivity in the treatment group is higher than that of the contrast group. The difference has much statistical significance. In the research, we put the 10 kinds of antibiotics commonly used in order according to the sensitivity high and low. The high sensitivity of the row was in front, and we artificially divided these ten kinds of drugs into two parts averagely. Then we made group two paired comparison of drug sensitivity in treatment group and control group. The comparison results are: $X^{2}=5.3252$ and $X^{2}=5.9260$, $\mathrm{P}<0.05$. The comparison results are in the following Table 1, Table 2.

Table 1. The comparison of the rate of relapse between treatment group and contrast group.

\begin{tabular}{ccc}
\hline Group & Cases (n) & Recurrence (n, \%) \\
\hline Control group & 52 & $27(51.92)$ \\
Treatment group & 63 & $16(25.39)$ \\
$X^{2}$ & & 8.5623 \\
P & \multicolumn{2}{c}{0.00779} \\
\hline
\end{tabular}

Table 2. The comparison of the drug susceptibility of 43 cases relapse sample.

\begin{tabular}{|c|c|c|c|c|c|c|}
\hline \multirow[b]{2}{*}{ Medicine } & \multicolumn{3}{|c|}{ Control group } & \multicolumn{3}{|c|}{ Treatment group } \\
\hline & Cases (n) & Sensitive (n, \%) & Resistance (n, \%) & Cases (n) & Sensitive (n, \%) & Resistance (n, \%) \\
\hline Minocycline & 27 & 23 (85.19) & $4(14.81)$ & 16 & 15 (93.75) & $1(6.25)$ \\
\hline Josamycin & 27 & $20(74.07)$ & 7 (25.93) & 16 & $14(87.50)$ & $2(12.50)$ \\
\hline Doxycycline & 27 & 19 (70.37) & 8 (29.63) & 16 & $14(87.50)$ & $2(12.50)$ \\
\hline Azithromycin & 27 & 17 (62.96) & $10(37.04)$ & 16 & $13(75.00)$ & $3(25.00)$ \\
\hline Thiamphenicol & 27 & $15(55.56)$ & $12(44.44)$ & 16 & $11(68.75)$ & $5(31.25)$ \\
\hline Roxithromycin & 27 & $14(51.85)$ & 13 (48.15) & 16 & $10(62.50)$ & $6(37.50)$ \\
\hline Clarithromycin & 27 & $13(48.15)$ & $14(51.86)$ & 16 & $10(62.50)$ & $6(37.50)$ \\
\hline Tetracycline & 27 & $12(44.44)$ & 15 (55.56) & 16 & $10(62.50)$ & $6(37.50)$ \\
\hline Cravit & 27 & $6(22.22)$ & $2 \mathrm{l}(77.78)$ & 16 & 7 (43.75) & $9(56.25)$ \\
\hline Sparfloxacin & 27 & $3(11.11)$ & 24 (88.89) & 16 & $5(31.25)$ & $11(68.75)$ \\
\hline
\end{tabular}


In Table 2, from the sensitive testing results of 10 common antibiotic medicines, we make comparison on the 5 drugs that are of higher sensitivity and another 5 drugs that are of lower sensitivity in the treatment group and contrast group on the basis of the principle of ratio principle, and $X X$ are 5.3252 and 5.9260, $\mathrm{P}<0.05$.

\section{Discussion}

From the result mentioned above, we can know, during the treatment of female genitourinary tract mycoplasma infection, the relapse rate of treatment group is lower than conventional group, which fully shows that the effect of intermittent medication treatment is much better. In addition, when the female patients with urogenital tract mycoplasma infection are cured with a traditional treatment, because of the side effects of drugs , they are not likely to take medicine that are required to, which makes the cure rate become lower and the relapse rate becomes higher. But intermittent medication is designed with the half-life period of medicine, which makes the medicine can be digested and absorbed easily, and the cure rate is high and the relapse rate is low [3] [4]. In this research we found a more effective method of the treatment on the female urogenital tract mycoplasma infection and showed the clinical significance in intermittent medication.

Genitourinary tract mycoplasma infection is a kind of rich man's disease at present society. It's caused by the sexually transmitted. Due to the opening characteristic of biorgan, especially the female urogenital tract, mycoplasma infection becomes more likely to be infectious, and this kind of problem brings great puzzles to human's lives which makes the treatment become more difficult. And if the medicine is taken inappropriate, it'll cause a vicious cycle. No matter how to treat, it is difficult to cure. In the study, we found that there are many cases because of the severe infection, infection both ureaplasma mycoplasma and mycoplasma hominis, commonly used antibiotics sensitivity is low at the same time, it is difficult to cure completely for the first time by conventional pharmacy, even using intermittent medication is still unable to cure. The main cause is that there's a close relationship between the absorption and use of drugs of patients themselves and multiple bacterial infections.

The study also showed that, treatment of female genitourinary tract mycoplasma infection by intermittent dosing, if patients relapse again, the result of drug susceptibility test sensitivity is higher, there is a significant difference compared to conventional pharmacy, $\mathrm{P}<0.05$, which suggests that intermittent dosing treatment of mycoplasma infection, the drug absorption and utilization degree is higher, and stronger sensitivity to drugs. Thus, during the further treatment more drugs will be used [5]. In the study we also found that, the proportion of drug medium sensitivity is higher. There are many possible factors to the cause: first the sample sources are different, or mycoplasma content is different, however, the result of the test method of the decision is related to the concentration of mycoplasma. That is, the high concentration and big content makes the deep color, otherwise light. Second the batch number of reagents is different. There are differences between the centration of package antibiotics; as a result, it shows different colors. Although some moderately sensitive results may cause due to the reasons above-mentioned, but it's still in accordance with clinical signs [5]. After further research, we found a more effective method of the treatment on the female urogenital tract mycoplasma infection and put forward the clinical significance in intermittent medication. And for better treatment effect on female urogenital tract mycoplasma Infection, we advise to use the intermittent medication treatment in clinical.

\section{Conclusion}

Intermittent dosing treatment has good effect on female genitourinary tract mycoplasma infection. When the female patients with urogenital tract mycoplasma infection are cured with a traditional treatment, because of the side effects of drugs, they are not likely to take medicines that are required to making the cure rate become lower and the relapse rate become higher. But intermittent medication is designed with the half-life period of medicine, which makes the medicine can be digested and absorbed easily, and the cure rate is high and the relapse rate is low. Therefore, this research of intermittent dosing treatment to female genitourinary tract mycoplasma infection can get much achievement in observing, guiding medication and prognosis judgment which has important clinical significance. Therefore, it's worth popularization and application.

\section{Study Limitations}

In this study, because of a shortage of research funding, the observation of the sample size is not so much, which 
was called small sample study. Although the achievement is outstanding, which is certain innovative, advanced, scientific and practical, the lack of large sample study, perhaps has certain limitation. We hope in the future we can do larger sample study under the support of some related departments. We are looking forward to making greater achievements.

\section{Acknowledgements}

During this topic research process, we got help from many departments and individuals, such as the city health bureau leadership and technology bureau leadership, and our hospital of obstetrics and gynecology, women's health, and other personnel not involved in this project research, all of them offered a great support and help in this research. Now here, all of members of this research group show our deepest appreciation to them, and wish them good health and everything goes well.

\section{Funding}

Guangxi Nanning City Science and Technology Plan Projects of the People's Republic of China (NO.: 201106048C-2).

\section{References}

[1] Vandepitte, J., Bukenya, J., Hughes, P., et al. (2012) Clinical Characteristics Associated with Mycoplasma Genitalium Infection among Women at High Risk of HIV and Other STI in Uganda. Sexually Transmitted Diseases, 39, $487-491$. http://dx.doi.org/10.1097/OLQ.0b013e31824b1cf3

[2] Vandepitte, J., Weiss, H.A., Kyakuwa, N., et al. (2013) Natural History of Mycoplasma Genitalium Infection in a Cohort of Female Sex Workers in Kampala, Uganda. Sexually Transmitted Diseases, 40, 422-427. http://dx.doi.org/10.1097/OLQ.0b013e31828bfccf

[3] Vandepitte, J., Weiss, H.A. and Bukenya, J. (2013) Alcohol Use, Mycoplasma Genitalium, and Other STIs Associated with HIV Incidence among Women at High Risk in Kampala, Uganda. Journal of Acquired Immune Deficiency Syndromes, 62, 119-126. http://dx.doi.org/10.1097/QAI.0b013e3182777167

[4] McGowin, C.L., Annan, R.S., Quayle, A.J., et al. (2012) Persistent Mycoplasma Genitalium Infection of Human Endocervical Epithelial Cells Elicits Chronic Inflammatory Cytokine Secretion. Infection and Immunity, 80, 3842-3849. http://dx.doi.org/10.1128/IAI.00819-12

[5] Vandepitte, J., Muller, E., Bukenya, J., et al. (2012) Prevalence and Correlates of Mycoplasma Genitalium Infection among Female Sex Workers in Kampala, Uganda. Journal of Infectious Diseases, 205, 289-296. http://dx.doi.org/10.1093/infdis/jir733 
Scientific Research Publishing (SCIRP) is one of the largest Open Access journal publishers. It is currently publishing more than 200 open access, online, peer-reviewed journals covering a wide range of academic disciplines. SCIRP serves the worldwide academic communities and contributes to the progress and application of science with its publication.

Other selected journals from SCIRP are listed as below. Submit your manuscript to us via either submit@scirp.org or Online Submission Portal.
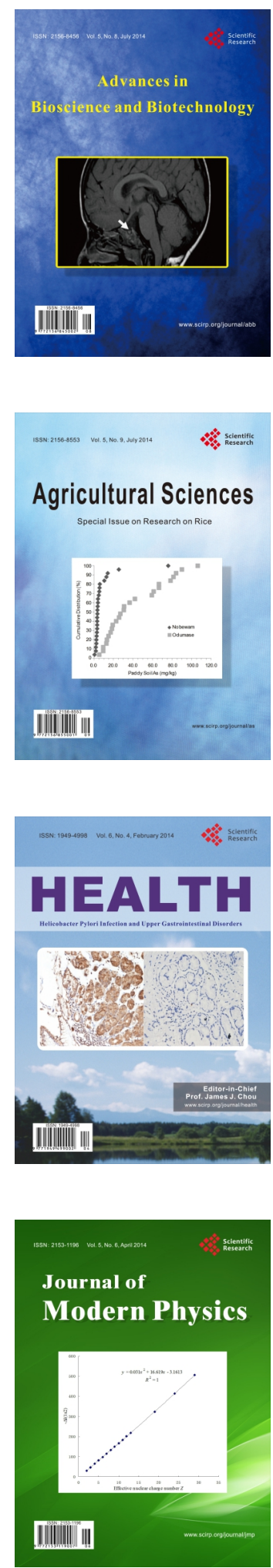
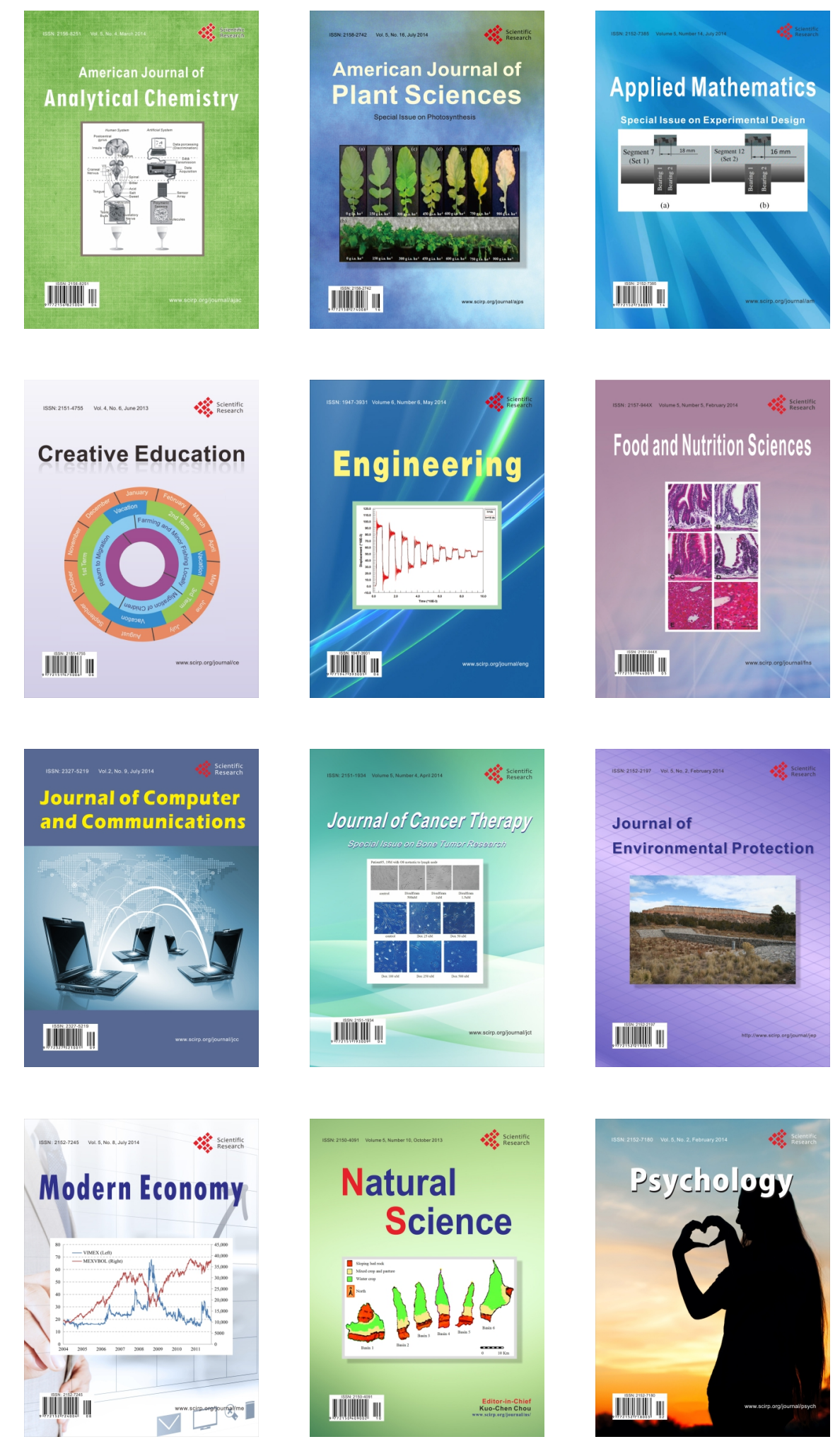